\title{
A miniaturized thermopneumatic infrared detector with capacitive read-out circuit
}

\author{
Marco Schossig, Volkmar Norkus, Gerald Gerlach \\ Technische Universität Dresden, Institute of Solid-State Electronics, 01062 Dresden \\ marco.schossig@tu-dresden.de
}

\begin{abstract}
In this paper we present an improved design of a miniaturized thermopneumatic infrared detector with capacitive read-out circuit. The hybrid assembly basically consists of a sealed cavity and a capacitive displacement transducer fabricated with silicon micromachining. The IR absorber is placed on a thin, free-standing silicon nitride membrane centrally arranged in the cavity. The very small capacitance changes are measured with an extremely sensitive 24-bit sigma-delta converter capable of detecting capacitance changes of a few aF. As a result, a responsivity of $714 \mathrm{aF} / \mu \mathrm{W}$ and a specific detectivity of $1.3 \cdot 10^{6} \mathrm{~cm} \mathrm{~Hz}^{1 / 2} \mathrm{~W}^{-1}$ were attained. Further improvements in performance can mainly be achieved by reducing the electrode gap of the capacitive displacement transducer.
\end{abstract}

Key words: infrared detector, thermal detector, Golay cell

\section{Introduction}

In biomimetics nature is used as a model for technical sensors. Humans and animals mainly rely on visual information and nature has developed a stunning diversity of sensor systems. For example, beetles of the genus Melanophila show pyrophilous ("fire-loving") behaviour and responses to infrared radiation (IR) stimuli (wavelength $(2.5 \ldots 4) \mu \mathrm{m})$ [1]. The IR organs of the Melanophila can be thought of as uncooled, miniaturized, fast, and highly sensitive IR sensors and they are termed as photomechanic IR receptors [2]. The IR perception of this insect is explained by a thermopneumatic model [3] as it is known from the Golay detector [4]. However, miniaturized Golay cell detectors are not commercially available up to now due to the limiting performance of miniature displacement transducers.

In [5] we reported on a first prototype of a miniaturized thermopneumatic infrared detector with capacitive read-out circuit. It showed a responsivity of a few $\mathrm{aF} / \mathrm{\mu W}$. The very small capacitance changes were measured with an extremely sensitive 24-bit sigma-delta converter capable of detecting capacitance changes of a few $a F$. The measured maximum specific detectivity was $4 \cdot 10^{4} \mathrm{~cm} \mathrm{~Hz}^{1 / 2} \mathrm{~W}^{-1}$. This is a quite low value but already one order of magnitude higher than other miniaturized thermopneumatic detectors with capacitive displacement transducer reported so far [6].
Here, we present an improved design of a miniaturized thermopneumatic infrared detector with capacitive read-out circuit and demonstrate that a capacitive detection is still very attractive. The IR sensor is characterised in terms of responsivity and specific detectivity.

\section{Sensor design and technology}

In principle, a thermopneumatic detector consists of a cavity that is filled with a gas (Fig. 1). The cavity is closed at one side by a flexible membrane. When the gas is illuminated by an IR source, the temperature rise makes the gas expand and causes the membrane to deflect. In our case, the membrane deflection is measured capacitively as a capacitance change of a capacitor. This capacitor consists of two parallel flat plates that are formed by the flexible membrane and the substrate material. For this purpose, the deflected membrane is covered with an electrode. The counter electrode is deposited onto a silicon (Si) or glass substrate. The electrode gap is defined by spacers made up of silicon nitride $\left(\mathrm{Si}_{3} \mathrm{~N}_{4}\right)$. The flexible membrane has a thickness of $1 \mu \mathrm{m}$ and is also made up of $\mathrm{Si}_{3} \mathrm{~N}_{4}$. In order to realize an excellent membrane flatness the membrane has a built-in tensile stress of $100 \mathrm{MPa}$. In our present prototype, the electrode gap of the capacitive displacement transducer has been reduced from $5 \mu \mathrm{m}$ to about $2.5 \mu \mathrm{m}$ and air is used as cavity gas. 
Fig. 1. Cross experimental prototype. section of an

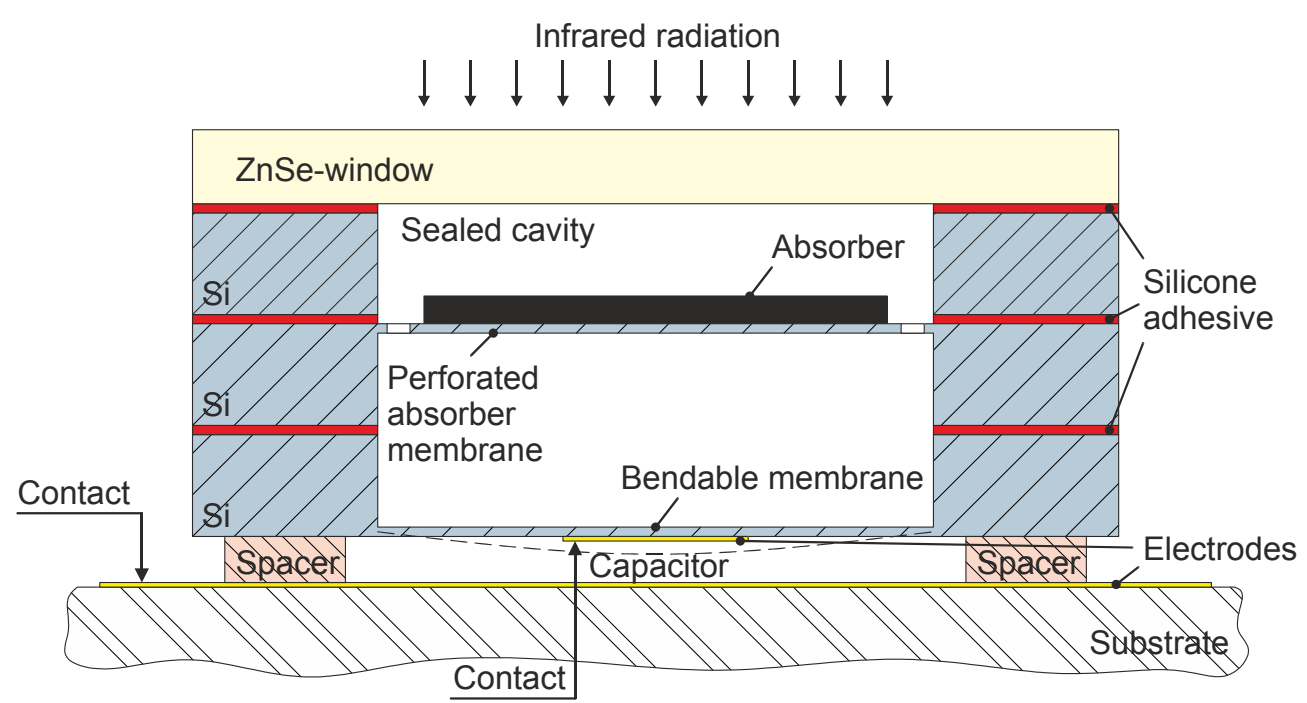

In order to absorb all incoming infrared radiation, an IR absorber with a very low thermal mass is placed onto a thin, freestanding, and perforated $\mathrm{Si}_{3} \mathrm{~N}_{4}$ membrane centrally arranged in the cavity. This absorber has a very high porosity of more than $90 \%$ which allows an immediately heating of the cavity gas. The central position of the absorber is necessary in order to achieve the best thermal isolation. As a result of this necessity, the cavity is made up of at least three so-called membrane chips, as shown in Fig. 2, that are bonded together with a silicone adhesive (cp. Fig. 1). Thereby, the lower part of the cavity is
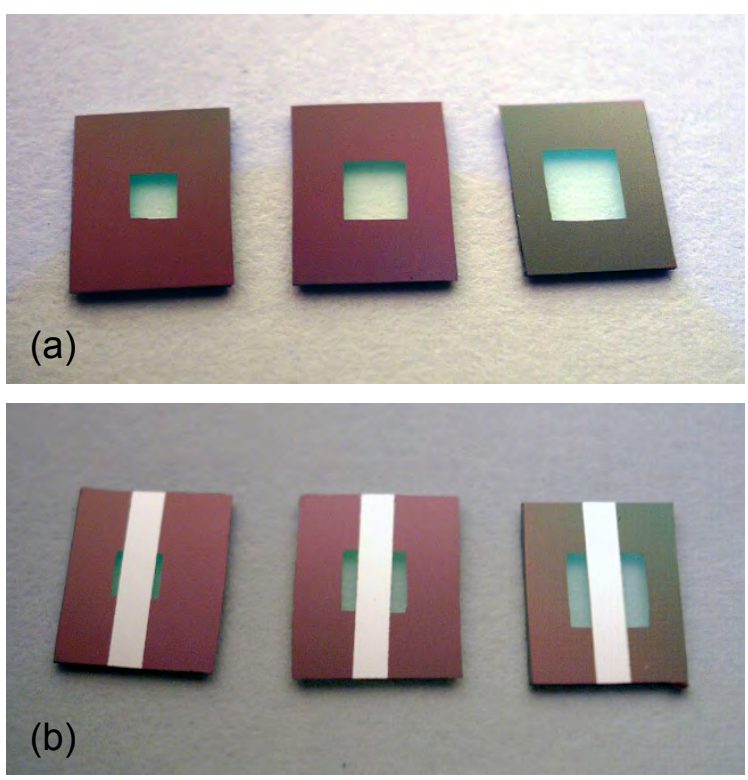

Fig. 2. Membrane chips fabricated by silicon micromachining with different membrane sizes of $(1.5 \times 1.5) \mathrm{mm}^{2}$, (2x2) $\mathrm{mm}^{2}$, and $(3 \times 3) \mathrm{mm}^{2}$, respectively, (a) without and (b) with electrode (electrode width: $1 \mathrm{~mm}$ ). made up of two membrane chips at which the upper membrane has been perforated by ion beam etching in order to connect the lower gas volume with the upper gas volume. The IR absorber is finally placed onto the perforated membrane. For the upper part of the cavity a membrane chip is used where the membrane has been removed. In order to improve the thermal isolation, a second chip can be bonded on top of it resulting in a higher cavity height. Finally, the cavity is closed by an IR transparent window that is bonded on top of the stack.

The fabrication of the membrane chips is done by silicon micromachining on a 4" Si-wafer with a thickness of $390 \mu \mathrm{m}$. In order to achieve membranes with a square shape, a dry plasma etching process is used together with $\mathrm{KOH}$ etching. The final size of the membrane chips is (5x6) $\mathrm{mm}^{2}$.

\section{Signal processing}

Some miniaturized Golay cell IR detectors have been developed using silicon micromachining technology together with a tunneling displacement transducer [7] as read-out mechanism. The main advantages of a capacitive detection system over a tunneling displacement transducer are its simple realisation and evaluation making it ideal for low-cost applications. Beyond, the low-frequency stability of tunneling displacement transducers is always uncertain and they show serious problems in terms of reliability [8].

There are many possibilities for the measurement of capacitance. The easiest are based on relaxation oscillators that measure the decay time of a $R C$ filter. But these methods do not have a sufficient high resolution and accuracy. 
In our prototype, we use a 24-bit capacitanceto-digital converter AD7745 from Analog Devices. This interface circuit is based on a sigma-delta core which directly converts a capacitance into a digital word, at high resolution $\left(2 \mathrm{aF} \mathrm{Hz}^{-1 / 2}\right)$, high linearity $( \pm 0.01 \%)$, and high accuracy $( \pm 4 \mathrm{fF})$ [9]. Such a sigmadelta converter consists of a switched-capacitor modulator, whose operation is based on balancing an unknown charge with a known reference charge of variable polarity, and a digital filter. All necessary functions such as sensor excitation, temperature sensor, voltage reference, etc. are included. This circuit has a 2-wire, $I^{2} \mathrm{C}$-compatible serial interface and allows assembly of the complete infrared detector together with signal evaluation into a TO-8 package (Fig. 3).

The resolution of the AD7745 is limited by noise which varies with selected conversion time $t_{\mathrm{C}}$ and excitation voltage $V_{\text {exc }}$ across the capacitance to be measured [5]. The conversion time defines the output data rate of the read-out circuit and, consequently, defines the maximum operation frequency of the detector. For the minimum conversion time of $11 \mathrm{~ms}$ the corresponding output data rate is $90.9 \mathrm{~Hz}$. This limits the maximum operation frequency of the detector to about $30 \mathrm{~Hz}$.

Environmental temperature variations can cause capacitor offsets which are commonly prevented by constructing a pneumatic leak between the sealed cavity and the capacitor. However, the AD7745 can be configured for differential mode allowing the use of a second, identically constructed but passive working Golay cell to avoid these influences without the need for a pneumatic leak.

\section{Experimental results and discussion}

The responsivity $R$ of the thermopneumatic infrared detector can be calculated by

$$
R=\frac{\Delta C}{P_{a b s}} .
$$

Here, $\Delta C$ denotes the measured capacitance change and $P_{\text {abs }}$ is the total IR power absorbed by the detector. Responsivity was measured with a $500 \mathrm{~K}$ black-body radiator of known irradiance $E$ at room temperature. Thereby, radiation was modulated by a mechanical chopper wheel. The total power absorbed by the detector is given by

$$
P_{a b s}=\alpha \cdot E \cdot A
$$

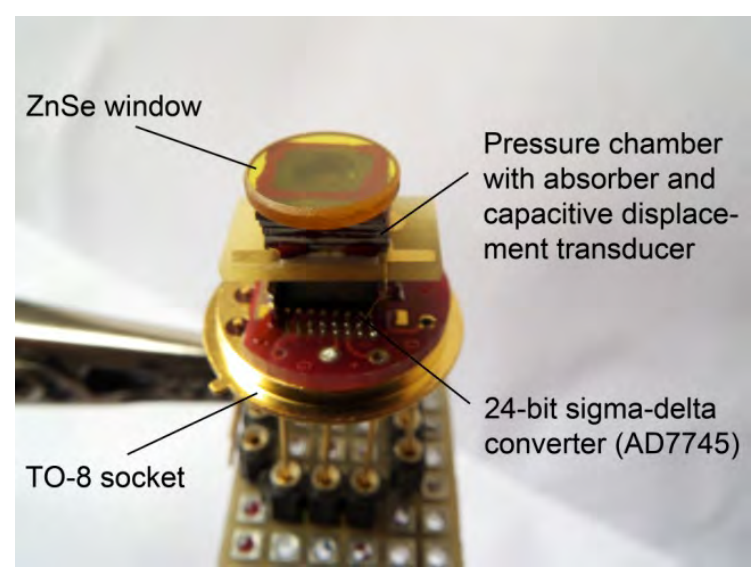

Fig. 3. Picture of the miniaturized thermopneumatic infrared detector assembled on a TO-8 socket.

with the radiation-sensitive area $A$ and the absorptance $\alpha$ of the detector. For the used IR absorber $\alpha$ is nearly unity and can, therefore, be neglected. As a result of the measurements, a responsivity of $714 \mathrm{aF} / \mu \mathrm{W}$ could be measured at low modulation frequencies $f<10 \mathrm{~Hz}$ (Fig. 4). This is an improvement of about two orders of magnitude compared to our first prototype [5].

For the determination of the specific detectivity $D^{*}$, the detector noise $N$ has to be taken into account:

$$
D^{*}=\frac{\sqrt{A} \cdot R}{N} .
$$

Noise measurements were performed in a sealed and shielded metal chamber to avoid environmental perturbations. As a result, an average noise $N$ of $116 \mathrm{aF} \mathrm{Hz}^{-1 / 2}$ could be measured. For the given detector area of $4.5 \mathrm{~mm}^{2}$, this results in a specific detectivity of about $1.3 \cdot 10^{6} \mathrm{~cm} \mathrm{~Hz}^{1 / 2} \mathrm{~W}^{-1}$ which is a moderate value.

In order to achieve an adequate value for the specific detectivity of $10^{8} \mathrm{~cm} \mathrm{~Hz}^{1 / 2} \mathrm{~W}^{-1}$, a responsivity of $55 \mathrm{fF} / \mu \mathrm{W}$ is needed when assuming the same noise value. Consequently, a further improvement in responsivity of about two orders of magnitude is necessary without increasing the noise.

A main improvement can be achieved with a reduction of the electrode gap $d_{\text {gap }}$ of the capacitive displacement transducer because responsivity $R$ is mainly influenced by $d_{\text {gap }}$ :

$$
R \propto \frac{1}{d_{g a p}^{2}} .
$$



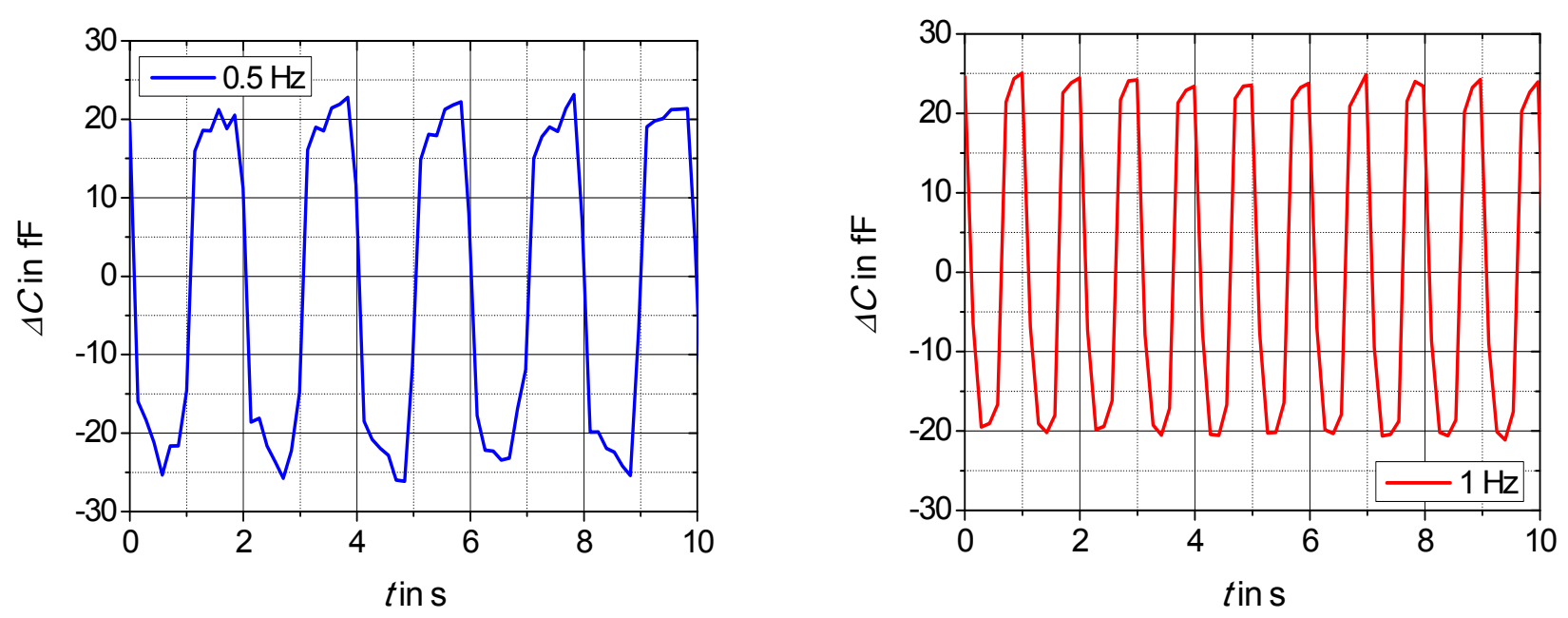

Fig. 4. Measured capacitance signal $\Delta C$ of the miniaturized thermopneumatic IR detector for modulation of $0.5 \mathrm{~Hz}$ and $1 \mathrm{~Hz}$, respectively. Measurement conditions: $500 \mathrm{~K}$ black-body radiator, irradiance of $14 \mu \mathrm{W} / \mathrm{mm}^{2}$, radiation-sensitive area $A=4.5 \mathrm{~mm}^{2}, \mathrm{ZnSe}$-window.

A first prototype with an electrode gap of less than $1 \mu \mathrm{m}$ showed a significant higher responsivity of about $21 \mathrm{fF} / \mu \mathrm{W}$ (Fig. 5). Unfortunately, there are serious problems in terms of reliability that have to be solved. Beyond that, noise seems to be increasing when reducing the electrode gap. However, it indicates that a much higher performance can be expected from miniaturized thermopneumatic IR detectors with capacitive displacement transducer. In order to achieve the required responsivity of $55 \mathrm{fF} / \mu \mathrm{W}$, an electrode gap of approximately $300 \mathrm{~nm}$ is necessary. This is quite challenging but feasible.

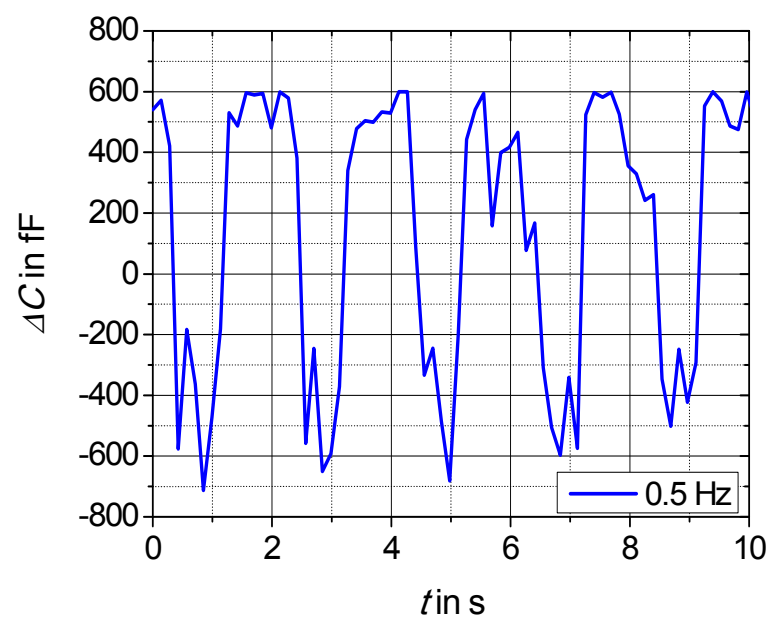

Fig. 5. Reduction of electrode gap $d_{\text {gap }}$ to less than $1 \mu \mathrm{m}$ promises a significant increase in performance. Measurement conditions: $500 \mathrm{~K}$ black-body radiator, irradiance of $14 \mu \mathrm{W} / \mathrm{mm}^{2}$, modulation frequency of $0.5 \mathrm{~Hz}$, radiation-sensitive area $A=3.8 \mathrm{~mm}^{2}, \mathrm{CaF}_{2}$ window.

\section{Conclusion and outlook}

A new concept for a miniaturized thermopneumatic IR detector with capacitive displacement transducer was presented. The measured detector performance of $R=714 \mathrm{aF} / \mu \mathrm{W}$ and $D^{*}=1.3 \cdot 10^{6} \mathrm{~cm} \mathrm{~Hz}^{1 / 2} \mathrm{~W}^{-1}$ is quite moderate but not limited by the read-out circuit. Compared to other miniaturized thermopneumatic infrared detectors with capacitive detection system, the attained performance is superior.

The used sigma-delta interface circuit offers a very attractive method for the precise measurement of very small capacitance changes that are directly converted into a digital word. The read-out circuit can be placed together with the IR detector into a small transistor housing and requires only four electrical connections, two for the $I^{2} \mathrm{C}$-compatible serial interface and two for the power supply.

In order to achieve an adequate value for the specific detectivity of $10^{8} \mathrm{~cm} \mathrm{~Hz}^{1 / 2} \mathrm{~W}^{-1}$, the responsivity of the thermopneumatic IR detector has to be further increased by about two orders of magnitude without increasing noise. This can be mainly achieved by reducing the electrode gap of the capacitive displacement transducer from actually $2.5 \mu \mathrm{m}$ to about $0.3 \mu \mathrm{m}$, which is quite challenging but feasible.

Finally, we could show that a capacitive detection system is still very attractive for a thermopneumatic infrared detector. Its simple realisation and evaluation as well as the possibility of manufacturing it by silicon micromachining technology make it ideal for low-cost applications. 


\section{Acknowledgement}

The authors would like to thank Dipl.-Ing. (FH) André Hiess and his colleagues from the Institute of semiconductors and Microsystems of TU Dresden for the fabrication of the membrane chips.

\section{References}

[1] W. G. Evans, Perception of infrared radiation from forest fires by melanophila acuminate de Geer (Buprestidae, Coleoptera), Ecology 47, 1061-1065 (1966); doi: 10.2307/1935658

[2] H. Schmitz, H. Bleckmann, The photomechanic infrared receptor for the detection of forest fires in the beetle Melanophila acuminata (Coleoptera: Buprestidae), Journal of Comparative Physiology 182(5), 647-657 (1998); doi: $10.1007 / \mathrm{s} 003590050210$

[3] W. G. Evans, Infrared radiation sensors of Melanophila acuminata (Coleoptera: Buprestidae): A thermopneumatic model, Annals of the Entomological Society of America 98(5), 738-746 (2005); doi: 10.1603/00138746(2005)098[0738:IRSOMA]2.0.CO;2

[4] M. J. E. Golay, A pneumatic infra-red detector, Review of Scientific Instruments 18(5), 357-362 (1947); doi: 10.1063/1.1740949

[5] M. Schossig, V. Norkus, G. Gerlach, A pneumatic infrared detector with capacitive read-out circuit, Proc. $12^{\text {th }}$ Int. Conf. Infrared Sensors and Systems (IRS $\left.{ }^{2}\right), 109-114$ (2011); doi: 10.5162/irs11/ip3

[6] K. Yamashita, A. Murata, M. Okuyama, Miniaturized infrared sensor using silicon diaphragm based on Golay cell, Sens. Act. A 66, 29-32 (1998); doi: 10.1016/S09244247(97)01702-0

[7] T. W. Kenny, J. K. Reynolds, Micromachined infrared sensors using tunneling displacement transducers, Rev. Sci. Instrum. 67 (1), pp. 112128, (1995); doi: 10.1063/1.1146559

[8] O. Ajakaiye, J. Grade, C. Shin, T. Kenny, Waferscale fabrication of infrared detectors based on tunneling displacement transducers, Sens. Act. A 134, pp. 575-581, (2007); doi: 10.1016/j.sna.2005.07.028

[9] J. O'Dowd, A. Callanan, G. Banarie, E. Company-Bosch, Capacitive sensor interfacing using sigma-delta techniques, IEEE Sensors 2005, pp. 951-954, (2005); doi: 10.1109/ICSENS.2005.1597858 